\title{
The Socioeconomic Impact of Hearing Loss in U.S. Adults
}

\author{
$* \uparrow$ Susan D. Emmett and *Howard W. Francis \\ *Department of Otolaryngology-Head and Neck Surgery, Johns Hopkins University School of Medicine; \\ †Department of International Health, Johns Hopkins Bloomberg School of Public Health, Baltimore, \\ Maryland, U.S.A.
}

Objective: To evaluate the associations between hearing loss and educational attainment, income, and unemployment/ underemployment in U.S. adults.

Study Design: National cross-sectional survey.

Setting: Ambulatory examination centers.

Patients: Adults aged 20 to 69 years who participated in the 1999 to 2002 cycles of the NHANES (National Health and Nutrition Examination Survey) audiometric evaluation and income questionnaire $(\mathrm{N}=3,379)$.

Intervention(s): Pure-tone audiometry, with hearing loss defined by World Health Organization criteria of bilateral puretone average of more than $25 \mathrm{~dB}(0.5,1,2,4 \mathrm{kHz})$.

Main Outcome Measure(s): Low educational attainment, defined as not completing high school; low income, defined as family income less than $\$ 20,000$ per year; and unemployment or underemployment, defined as not having a job or working less than 35 hours per week.

Results: Individuals with hearing loss had 3.21 times higher odds of low educational attainment $(95 \%$ confidence interval
[95\% CI], 2.20-4.68) compared with normal-hearing individuals. Controlling for education, age, sex, and race, individuals with hearing loss had 1.58 times higher odds of low income $(95 \% \mathrm{CI}$, 1.16-2.15) and 1.98 times higher odds of being unemployed or underemployed (95\% CI, 1.38-2.85) compared with normalhearing individuals.

Conclusion: Hearing loss is associated with low educational attainment in U.S. adults. Even after controlling for education and important demographic factors, hearing loss is independently associated with economic hardship, including both low income and unemployment/underemployment. The societal impact of hearing loss is profound in this nationally representative study and should be further evaluated with longitudinal cohorts. Received institutional review board approval (National Center for Health Statistics Institutional Review Board Protocol no. 98-12). Key Words: Hearing loss_Epidemiology_Nutrition surveys-Socioeconomic status-United States.

Otol Neurotol 36:545-550, 2015.
The World Health Organization (WHO) estimates that hearing loss affects 360 million people worldwide (1). Hearing impairment ranked as the 13th highest contributor to the global burden of disease in 2002 and is projected to be the ninth leading contributor worldwide and the seventh in high-income countries by 2030 (2). In the United States alone, $12.7 \%$ of individuals older than 12 years are estimated to be affected (3). Given the prominence of hearing loss both globally and domestically, understanding its societal impact is essential.

Address correspondence and reprint requests to Susan D. Emmett, M.D., Department of Otolaryngology-Head and Neck Surgery, Johns Hopkins University School of Medicine, 601 North Caroline St., 6th Floor, Baltimore, MD 21287, U.S.A.; E-mail: semmett2@jhmi.edu

The authors disclose no conflicts of interest.

This study was supported by T32 Research Training Grant 5T32DC000027-25 from the National Institutes of Health.

This work was presented at the American Otological Society 147th Annual Meeting in Las Vegas, NV, U.S.A., on May 17, 2014.
We have long recognized that severe to profound losses have a tremendous impact on education and employment (4-6). It is now becoming clear that even mild losses can adversely affect speech and language development and school performance in childhood (7-11). Data from Finland suggest that decreased school performance early in life in individuals with hearing loss continues to have an adverse effect into young adulthood, with a higher likelihood of not qualifying for intermediate or higher education and more frequent unemployment (12). These data highlight the importance of examining the societal impact of hearing loss from a broader perspective, using population-based methods to assess the socioeconomic consequences of all levels of hearing loss.

We have therefore evaluated the associations between hearing loss and low educational attainment, low income, and unemployment/underemployment using the 1999 to 2000 and 2001 to 2002 cycles of the National Health and 
Nutrition Examination Survey (NHANES), a nationally representative cross-sectional survey that evaluates the health of the civilian noninstitutionalized U.S. population. By pursuing this study, we seek to assess the relationship between socioeconomic status and hearing loss in the United States.

\section{MATERIALS AND METHODS}

\begin{abstract}
Study Cohort
The NHANES is an ongoing nationally representative crosssectional survey that assesses the health of the civilian noninstitutionalized U.S. population. Every 2 years, a complex multistage probability sampling design is used to select a sample of the population for interview and examination. Low-income individuals, racial minorities, and older adults are oversampled. Sampling weights are used to account for this complex design, yielding results that are generalizable to the U.S. population (13).

We used the 1999 to 2000 and 2001 to 2002 cycles of NHANES, which collected audiometric data and income information on adults aged 20 to 69 years. These cycles were combined to analyze a total of 4 years of data based on the National Center for Health Statistics (NCHS) recommendations (13). One-half of all participants aged 20 to 69 years were selected to participate in audiometric testing. Reported annual family income was required for inclusion in the study cohort $(\mathrm{n}=157$ excluded), yielding a final sample size for the analysis of $\mathrm{N}=3,379$.
\end{abstract}

\section{Audiometric Assessment}

Audiometry was performed by trained examiners in a dedicated sound-isolating room in the mobile examination center according to established NHANES protocols (14). Briefly, airconduction thresholds were obtained from 0.5 to $8 \mathrm{kHz}$ over an intensity range of -10 to $120 \mathrm{~dB}$ using an Interacoustics Model AD226 audiometer with automated and/or manual testing per protocol. Stimuli were presented through supra-aural headphones (TDH-39) except in cases of ear canal collapse or crossover retesting, when insert earphones (Ear Tone 3A) were used. Equipment was calibrated daily, and ambient noise levels were monitored with a sound meter.

The 1-kHz threshold was tested twice in each ear, and audiometry was repeated if a greater than $10-\mathrm{dB}$ discrepancy was observed. Crossover retesting for asymmetric hearing loss was performed whenever thresholds differed between ears by $25 \mathrm{~dB}$ $(0.5,1 \mathrm{kHz})$ or $40 \mathrm{~dB}(\geq 2 \mathrm{kHz})$. Masking was not performed. Hearing loss was defined by WHO criteria of pure-tone average greater than $25 \mathrm{~dB}(0.5,1,2$, and $4 \mathrm{kHz})$ in both ears (15). The first $1-\mathrm{kHz}$ threshold and manual retesting thresholds, when performed, were used in all analyses.

\section{Demographic and Socioeconomic Variables}

Demographic and socioeconomic data were obtained from interviews. Race/ethnicity was categorized as non-Hispanic white (white), non-Hispanic black (black), Mexican-American/ other Hispanic (Hispanic), or other. Low educational attainment was defined by the National Center for Education Statistics classification of not completing high school (15). Low income was defined as family income less than $\$ 20,000$ per year (16). Employment was assessed using a modified Bureau of Labor Statistics' definition combining unemployment and underemployment, defined as not having a job or working less than 35 hours per week (17).

\section{Analysis}

Sample weights were used to account for a complex sampling design according to NCHS guidelines in all analyses except for Table 1 (13). The purpose of Table 1 was to provide descriptive statistics of the study cohort instead of nationally generalizable estimates, and therefore weights were excluded. Demographic and socioeconomic characteristics were compared by hearing status using the $\chi^{2}$ test. Simple and multiple logistic regression analyses were used to evaluate the associations between hearing loss and low education, low income, and unemployment/ underemployment. Adequate fit of multiple logistic regression models was assessed using the Archer and Lemeshow $F$-adjusted mean residual test (18). Odds ratios (ORs) reported in the text are from multiple logistic regression models unless otherwise stated. The Taylor Series Linearization method for variance estimation was used per NCHS guideline (13). All analyses were conducted using STATA 12.1 (StataCorp, College Station, TX, USA). Two-sided values of $p<0.05$ were considered statistically significant.

\section{Ethics}

Consent was obtained from all participants. Study protocols were approved by the NCHS Institutional Review Board (Protocol no. 98-12).

\section{RESULTS}

\section{Study Cohort}

There were notable demographic and socioeconomic differences between individuals with hearing loss $(n=318)$ and those with normal hearing $(\mathrm{n}=3,061)$ in this cohort (Table 1). Individuals with hearing loss tended to be male $(70 \%$ versus $45 \% ; p<0.001)$, aged 45 years or older (90\% versus $41 \% ; p<0.001)$, and were less likely to be black $(12 \%$ versus $20 \% ; p=0.01)$ than normal-hearing individuals. Socioeconomically, individuals with hearing loss were more likely to have not completed high school (48\% versus $28 \%$; $p<0.001$ ), have an annual income less than $\$ 20,000(38 \%$ versus $28 \% ; p=0.001)$, and be unemployed or underemployed (62\% versus $45 \%$; $p<0.001)$.

TABLE 1. Demographic and socioeconomic characteristics by hearing status of participants with both audiometric and income data, NHANES 1999 to 2002, $N=3,379^{a}$

\begin{tabular}{lccr}
\hline Characteristic & $\begin{array}{c}\text { Normal hearing } \\
(\mathrm{n}=3,061)\end{array}$ & $\begin{array}{c}\text { Hearing loss } \\
(\mathrm{n}=318)\end{array}$ & $p$ value \\
\hline Male & $1,363(45)$ & $222(70)$ & $<0.001$ \\
Age $\geq 45$ years & $1,247(41)$ & $285(90)$ & $<0.001$ \\
Race/ethnicity & & & 0.01 \\
$\quad$ White & $1,437(47)$ & $167(53)$ & \\
Black & $614(20)$ & $39(12)$ & \\
Hispanic & $918(30)$ & $102(32)$ & \\
$\quad$ Other & $92(3)$ & $10(3)$ & \\
Education $<$ high school $_{\text {Income }<\$ 20,000}$ & $863(28)$ & $153(48)$ & $<0.001$ \\
Unemployed or underemployed $^{c}$ & $871(28)$ & $120(38)$ & 0.001 \\
\hline
\end{tabular}

${ }^{a}$ All values are $\mathrm{n}(\%)$.

${ }^{b}$ Defined as bilateral pure-tone average $>25 \mathrm{~dB}(0.5,1,2$, and $4 \mathrm{kHz})$.

${ }^{c}$ Not having a job or working less than 35 hours per week. 


\section{Low Educational Attainment}

Low educational attainment, defined as not completing high school (15), was associated with race/ethnicity and hearing status (Table 2). Black and Hispanic individuals had more than three times higher odds of low educational attainment (OR, 3.44; 95\% confidence interval [95\% CI], 2.62-4.53; OR, 6.14; 95\% CI, 4.83-7.80, respectively) than white individuals. Individuals with hearing loss had more than three times higher odds of low educational attainment (OR, 3.21; 95\% CI, 2.20-4.68) than normalhearing individuals. These associations remained significant and of similar magnitude after controlling for sociodemographic factors (sex, age, and race). Sex and age were not associated with education.

\section{Low Income}

Low income, defined as an annual family income less than $\$ 20,000$ (16), was associated with multiple sociodemographic factors and hearing status (Table 3). Black and Hispanic individuals had more than two times higher odds of having a low income (OR, 2.45; 95\% CI, 1.84-3.27; OR, 2.19; 95\% CI, 1.44-3.33, respectively) than white individuals. Individuals with low educational attainment had three times higher odds of having a low income (OR, 3.10; 95\% CI, 2.33-4.14) than those who had completed a high school education or greater. In addition, individuals with hearing loss demonstrated 1.5 times higher odds of having a low income $(1.58 ; 95 \%$ CI, 1.16-2.15) than individuals with normal hearing. Men and individuals aged 45 years or older were less likely to report having a low income $(0.65 ; 95 \% \mathrm{CI}$, $0.56-0.75$; OR, $0.65 ; 95 \%$ CI, 0.54-0.77, respectively). All associations remained significant and of similar magnitude after controlling for sociodemographic factors, including education.

TABLE 2. Unadjusted and adjusted odds ratios and 95\% confidence intervals of low educational attainment (less than high school) by sociodemographic characteristics and hearing

\begin{tabular}{|c|c|c|c|c|}
\hline \multirow[b]{2}{*}{ Characteristic } & \multicolumn{2}{|c|}{ Unadjusted } & \multicolumn{2}{|l|}{ Adjusted $^{a}$} \\
\hline & OR $(95 \% \mathrm{CI})$ & $p$ value & OR $(95 \% \mathrm{CI})$ & $p$ value \\
\hline \multicolumn{5}{|l|}{ Sex } \\
\hline Female & Reference & & Reference & \\
\hline Male & $1.06(0.87-1.30)$ & 0.56 & $0.95(0.77-1.18)$ & 0.65 \\
\hline \multicolumn{5}{|l|}{ Age (yr) } \\
\hline$<45$ & Reference & & Reference & \\
\hline$\geq 45$ & $1.08(0.87-1.34)$ & 0.46 & $1.09(0.84-1.41)$ & 0.52 \\
\hline \multicolumn{5}{|l|}{ Race/ethnicity } \\
\hline White & Reference & & Reference & \\
\hline Black & $3.10(2.39-4.02)$ & $<0.001$ & $3.44(2.62-4.53)$ & $<0.001$ \\
\hline Hispanic & $5.63(4.49-7.06)$ & $<0.001$ & $6.14(4.83-7.80)$ & $<0.001$ \\
\hline Other & $1.52(0.92-2.50)$ & 0.10 & $1.56(0.92-2.64)$ & 0.10 \\
\hline \multicolumn{5}{|l|}{ Hearing status } \\
\hline Normal hearing & g Reference & & Reference & \\
\hline Hearing loss & $2.61(1.95-3.49)$ & $<0.001$ & $3.21(2.20-4.68)$ & $<0.001$ \\
\hline
\end{tabular}

${ }^{a}$ Adjusted for all variables in the table.
TABLE 3. Unadjusted and adjusted odds ratios of low income (annual family income less than \$20,000) by sociodemographic characteristics and hearing status

\begin{tabular}{|c|c|c|c|c|}
\hline \multirow[b]{2}{*}{ Characteristic } & \multicolumn{2}{|c|}{ Unadjusted } & \multicolumn{2}{|l|}{ Adjusted $^{a}$} \\
\hline & OR $(95 \% \mathrm{CI})$ & $p$ value & OR $(95 \% \mathrm{CI})$ & $p$ value \\
\hline \multicolumn{5}{|l|}{ Sex } \\
\hline Female & Reference & & Reference & \\
\hline Male & $0.71(0.61-0.81)$ & $<0.001$ & $0.65(0.56-0.75)$ & $<0.001$ \\
\hline \multicolumn{5}{|c|}{ 10.0.0 } \\
\hline$<45$ & Reference & & Reference & \\
\hline$\geq 45$ & $0.69(0.59-0.81)$ & $<0.001$ & $0.65(0.54-0.77)$ & $<0.001$ \\
\hline \multicolumn{5}{|l|}{ Race/ethnicity } \\
\hline White & Reference & & Reference & \\
\hline Black & $2.98(2.24-3.97)$ & $<0.001$ & $2.45(1.84-3.27)$ & $<0.001$ \\
\hline Hispanic & $3.16(2.25-4.43)$ & $<0.001$ & $2.19(1.44-3.33)$ & 0.001 \\
\hline Other & $1.79(0.98-3.28)$ & 0.06 & $1.69(0.93-3.08)$ & 0.08 \\
\hline \multicolumn{5}{|l|}{ Education } \\
\hline$\geq$ High school & Reference & & Reference & \\
\hline$<$ High school & $3.87(3.08-4.87)$ & $<0.001$ & $3.10(2.33-4.14)$ & $<0.001$ \\
\hline \multicolumn{5}{|l|}{ Hearing status } \\
\hline Normal hearing & Reference & & Reference & \\
\hline Hearing loss & $1.30(1.01-1.68)$ & 0.04 & $1.58(1.16-2.15)$ & 0.005 \\
\hline
\end{tabular}

${ }^{a}$ Adjusted for all variables in the table.

\section{Unemployment and Underemployment}

Unemployment or underemployment, characterized by the modified Bureau of Labor Statistics' definition of not having a job or working less than 35 hours per week (17), was associated with age, education, and hearing status (Table 4). Individuals aged 45 years or older and those with low educational attainment had higher odds of being unemployed or underemployed (OR, 1.32; 95\% CI, 1.10-1.59; OR, 1.92; 95\% CI, 1.52-2.44, respectively). Individuals with hearing loss had nearly two times higher odds of unemployment or underemployment (OR, 1.98; $95 \% \mathrm{CI}, 1.38-2.85)$ than individuals with normal hearing.

TABLE 4. Unadjusted and adjusted odds ratios of unemployment or underemployment (not working or working for less than 35 hours per week) by sociodemographic characteristics and hearing status

\begin{tabular}{|c|c|c|c|c|}
\hline \multirow[b]{2}{*}{ Characteristic } & \multicolumn{2}{|l|}{ Unadjusted } & \multicolumn{2}{|l|}{ Adjusted $^{a}$} \\
\hline & OR $(95 \% \mathrm{CI})$ & $p$ value & OR $(95 \% \mathrm{CI})$ & $p$ value \\
\hline \multicolumn{5}{|l|}{ Sex } \\
\hline Female & Reference & & Reference & \\
\hline Male & $0.30(0.25-0.37)$ & $<0.001$ & $0.28(0.23-0.34)$ & $<0.001$ \\
\hline \multicolumn{5}{|l|}{ Age (yr) } \\
\hline$<45$ & Reference & & Reference & \\
\hline$\geq 45$ & $1.47(1.22-1.78)$ & $<0.001$ & $1.32(1.10-1.59)$ & 0.005 \\
\hline \multicolumn{5}{|l|}{ Race/ethnicity } \\
\hline White & Reference & & Reference & \\
\hline Black & $1.28(1.03-1.58)$ & 0.03 & $1.17(0.93-1.48)$ & 0.17 \\
\hline Hispanic & $1.00(0.79-1.27)$ & 0.98 & $0.90(0.65-1.23)$ & 0.50 \\
\hline Other & $1.24(0.67-2.32)$ & 0.48 & $1.15(0.60-2.21)$ & 0.66 \\
\hline \multicolumn{5}{|l|}{ Education } \\
\hline$\geq$ High school & Reference & & Reference & \\
\hline$<$ High school & $1.85(1.53-2.24)$ & $<0.001$ & $1.92(1.52-2.44)$ & $<0.001$ \\
\hline \multicolumn{5}{|l|}{ Hearing status } \\
\hline Normal hearing & Reference & & Reference & \\
\hline Hearing loss & $1.77(1.26-2.47)$ & 0.002 & $1.98(1.38-2.85)$ & 0.001 \\
\hline
\end{tabular}

${ }^{a}$ Adjusted for all variables in the table. 
Men were less likely to be unemployed or underemployed (OR, 0.28; 95\% CI, 0.23-0.34). Race/ethnicity was not associated with unemployment or underemployment. All associations again remained significant and of similar magnitude after adjustment for sociodemographic factors, including education.

\section{DISCUSSION}

In this nationally representative study of U.S. adults aged 20 to 69 years, hearing loss was independently associated with low educational attainment, low income, and unemployment/underemployment. The associations between hearing loss and both income and employment status remained significant even after accounting for the low educational attainment observed in the hearingimpaired population. These findings suggest that hearing loss has substantial socioeconomic implications. There are few prior studies investigating the socioeconomic impact of hearing loss. Our results are consistent with populationbased studies from Norway (19), Sweden (20), Finland (12), and Australia (21), contributing valuable U.S. data to this small but growing literature. Importantly, our study represents one of four in the world using standardized audiometric testing instead of self-report to define hearing loss $(12,19,22)$. The only other nationally representative survey on the topic in the United States relied on a coded diagnosis of hearing loss to identify affected individuals (23). The strengths of our study therefore include use of standardized audiometric testing and an adjudicated definition of hearing loss from the WHO in the context of a nationally representative sample.

The results of our study reveal notable demographic differences in the U.S. population with hearing loss. Individuals with hearing loss were more likely to be male, aged 45 years or older, and less likely to be black. Male sex and increasing age are known nonmodifiable risk factors for hearing loss that have been elucidated in other studies $(20,24-26)$. Black race is consistently associated with a $60 \%$ to $70 \%$ decreased odds of hearing loss in observational studies $(24,26-28)$. Lin and colleagues (28) suggest that skin color, and by extension melanin levels in the cochlea, may be the mechanism behind this protective association.

In our study, low educational attainment was associated with race and hearing status. Black and Hispanic individuals had greater than three and six times the odds of not completing high school, respectively, compared with white individuals. The Current Population Survey conducted by the U.S. Census Bureau and the Bureau of Labor Statistics corroborate this racial disparity, reporting a significant difference based on race in the proportion of U.S. adults who have not completed high school (29). The association between hearing loss and low educational attainment has not previously been studied in the United States to our knowledge. Our study suggests that individuals with hearing loss have a higher likelihood of low educational attainment, which is consistent with the
Finnish longitudinal cohort of young adults of Järvelin et al. (12) in which individuals with hearing loss were at more than two times higher odds of not continuing from secondary to higher education. A Norwegian-based study found a similar association between hearing loss and not completing high school (19). Aside from the influence of race and hearing loss, other evidence suggests a difference in educational attainment based on sex (29). Our study did not demonstrate a sex-based disparity in low educational attainment, however. Both the Current Population Survey and NHANES are based on self-report and are thus subject to recall and social desirability bias, which may explain, in part, the discrepancies in results.

Low income was associated with multiple factors in our study, including race/ethnicity, sex, age, educational attainment, and hearing status. Racial disparities in low income are well documented in other studies, with nonHispanic blacks and Hispanics significantly more likely to report having a low income than non-Hispanic whites $(29,30)$. The U.S. Current Population Survey corroborates the sex- and age-related differences observed in our study, reporting that women and younger age groups are significantly more likely to report having a low income (29). Low educational attainment is another wellestablished risk factor for low income described elsewhere in existing literature $(15,29,30)$. In our study, hearing loss increased the likelihood of low income more than 1.5 -fold. Income was not directly addressed in the international articles on socioeconomic status and hearing loss. In the only U.S. article on the topic to date, Jung and Bhattacharyya (23) noted that individuals with a coded diagnosis of hearing loss were 2.5 times less likely to report any wage income than those without hearing loss, and the mean wage income for individuals with hearing loss was nearly $\$ 8,000$ less than the mean wage of the population without hearing loss.

Lastly, our study revealed associations between unemployment/underemployment and age, educational attainment, sex, and hearing status. The Centers for Disease Control and Prevention report similar discrepancies in age and educational attainment, with a significantly higher prevalence of unemployment among younger age groups and those who did not complete a high school education (31). Multiple international studies corroborate the association between hearing loss and unemployment/underemployment $(12,20,21)$, along with Jung and Bhattacharyya (23) domestically. It is important to note that our study did not reveal racial differences in employment/underemployment status. This finding conflicts with those of other sources, such as the Centers for Disease Control's Behavioral Risk Factor Surveillance System (BRFSS), which found a twofold higher prevalence of unemployment among non-Hispanic blacks compared with non-Hispanic whites (31). There are a few possible explanations for this discrepancy. The BRFSS does not consider underemployment in its definition of unemployment, and the survey excludes certain populations, including individuals without landline telephones and those who are homeless (31). Both the BRFSS and NHANES rely on self-report, raising the risk of recall bias and social 
desirability bias. The BRFSS had a low median state response rate (51.4\%), which also increases the risk of nonresponse bias (31). However, given that discrepancies were observed between our study and other established economic data sources with regard to both race in unemployment and sex in educational attainment, it is possible that underreporting of socioeconomic information in NHANES could be responsible.

Our study highlights important racial and sex-based socioeconomic disparities that have been described in the wider disparities literature. Notably, it also emphasizes hearing loss as another potential source of socioeconomic disparity. In this study, hearing loss was the only factor that was independently associated with all three measures of socioeconomic status: low educational attainment, low income, and unemployment/underemployment. The mechanisms for these associations are not well defined. Considering possible pathways from existing literature, Järvelin et al. (12) postulates that the lower levels of education associated with hearing loss is the likely driver behind a higher likelihood of unemployment in this population. The independent effect of hearing loss on unemployment/underemployment after controlling for education in our study suggests that education alone is not the only mechanism through which hearing loss is associated with employment status. Kramer et al. (32) proposes a possible non-education-related pathway for this association in a Dutch survey of 150 hearing-impaired individuals and 60 job-matched normally hearing controls. In this study, hearingimpaired individuals reported significantly less "control" in the workplace, higher effort required during listening, and more frequent sick leave because of distress than their normal-hearing colleagues.

There are alternative explanations for the relationship between socioeconomic status and hearing loss that should be considered. Low socioeconomic status itself could be the driver behind the development of hearing loss, particularly if less-educated individuals pursue types of work that increase their exposure to noise. Other alternative explanations include higher levels of recreational noise exposure and increased perinatal risk factors in lower socioeconomic groups. In a large survey of Dutch adolescents aged 12 to 19 years, Vogel et al. (33) observed a difference in risk-taking behavior related to high-volume music on MP3 players based on socioeconomic status. In considering early-life effects of socioeconomic status, there are substantial data demonstrating an association between a low socioeconomic status and an intrauterine growth restriction, which places children at an increased risk of both perinatal complications that may be associated with hearing loss and cognitive delays (34). In a retrospective cohort of greater than 300,000 children entering kindergarten in Florida, Resnick et al. (35) observed that sociodemographic predictors, including poverty, low maternal education, and nonwhite race, substantially raised the risk of mild educational disabilities. Because of their high prevalence, the attributable impact of each of these sociodemographic predictors on adverse educational outcomes was between five and 10 times greater than the effect of a low birth weight alone (35).
The design of NHANES limited our ability to assess potential mediators in the relationship between hearing loss and socioeconomic status. Its cross-sectional nature does not allow for assessment of temporality in mediating relationships, and we were further restricted to exploring medical conditions and environmental exposures that were collected as a part of the study. We nevertheless evaluated available known risk factors for hearing loss as potential mediators in the socioeconomic status relationship, including diabetes, hypertension, body mass index, smoking, and noise exposure from occupational and nonoccupational sources (36-39). None of these medical conditions or environmental exposures demonstrated statistical mediation in our study (data not shown). The relationship between socioeconomic status and hearing loss is undoubtedly complex, likely involving multiple simultaneous pathways. Future longitudinal studies will be critical to better understand the mechanisms behind these associations.

There are notable weaknesses in our study. During data collection on underemployment, the NHANES protocol did not differentiate between individuals who work 35 hours or less per week because of choice and those who work part time because of not being able to find full-time employment. The absence of this distinction required us to use a modification of the Bureau of Labor Statistics definition of underemployment, which could have led to an overinflation of unemployment/underemployment in our study. Because of the nature of the income information released in NHANES, we were unable to assess the numeric difference in income between the hearing-impaired and normal-hearing populations. The NHANES participants have a choice of reporting their income based on a screening question of more than or less than $\$ 20,000$ or reporting an exact figure, with deidentified income data then released in $\$ 5,000$ to $\$ 10,000$ increments. A substantial number of participants chose to answer only the screening question, and thus, to maximize the number of eligible participants, we analyzed the available income data using the screening cut point of $\$ 20,000$. Reliance on this screening question prevented us from being able to use the standard income-to-poverty ratio definition of low income that incorporates number and age of individuals in the household (29). In future studies, more precise information on the differences in income would be extremely valuable for evaluating the economic cost of hearing loss.

Cross-sectional surveys such as NHANES are by nature unable to assess temporality, and thus we were unable to conclude causation in the observed associations between hearing loss and socioeconomic status. Longitudinal studies will be essential for better delineation of this relationship and improved understanding of the underlying mechanisms. Our study was also not equipped to investigate effective interventions to reduce the impact of hearing loss on education, income, and employment. It is possible that consistent hearing aid use is able to reduce the impact of hearing loss on one or all of the measures of socioeconomic status we explored. The number of individuals using hearing aids in this NHANES cohort was too small to evaluate this effect, however. A priority 
in future research should be investigation of successful strategies to reduce or, ideally, ameliorate the untoward socioeconomic effects of hearing loss.

With $5 \%$ of the world's population experiencing hearing loss, understanding and quantifying its societal impact are critical. This study contributes important data to the small but growing literature suggesting that hearing loss has an adverse effect on educational attainment, income, and employment status. Further longitudinal studies are urgently needed to better define these relationships and provide possible solutions for reducing this important disparity.

\section{REFERENCES}

1. World Health Organization. Deafness and hearing loss. Available at: http://www.who.int/mediacentre/factsheets/fs300/en/. Accessed December 3, 2013.

2. Mathers CD, Loncar D. Projections of global mortality and burden of disease from 2002 to 2030. PLoS Med 2006;3:e442.

3. Lin FR, Niparko JK, Ferrucci L. Hearing loss prevalence in the United States. Arch Intern Med 2011;171:1851-2.

4. Blanchfield BB, Feldman JJ, Dunbar JL, et al. The severely to profoundly hearing-impaired population in the United States: prevalence estimates and demographics. J Am Acad Audiol 2001;12:183-9.

5. Mohr PE, Feldman JJ, Dunbar JL, et al. The societal costs of severe to profound hearing loss in the United States. Int J Technol Assess Health Care 2000;16:1120-35.

6. Winn S. Employment outcomes for people in Australia who are congenitally deaf: has anything changed? Am Ann Deaf 2007;152:382-90.

7. Moeller MP. Early intervention and language development in children who are deaf and hard of hearing. Pediatrics 2000;106:E43.

8. Kennedy CR, McCann DC, Campbell MJ, et al. Language ability after early detection of permanent childhood hearing impairment N Engl J Med 2006;354:2131-41.

9. Khairi Md, Daud M, Noor RM, et al. The effect of mild hearing loss on academic performance in primary school children. Int $J$ Pediatr Otorhinolaryngol 2010;74:67-70.

10. Bess FH, Dodd-Murphy J, Parker RA. Children with minimal sensorineural hearing loss: prevalence, educational performance, and functional status. Ear Hear 1998;19:339-54

11. Blair JC. The effects of mild sensorineural hearing loss on academic performance of young school-age children. Volta Rev 1985;87:87-93.

12. Järvelin MR, Mäki-Torkko E, Sorri MJ, et al. Effect of hearing impairment on educational outcomes and employment up to the age of 25 years in northern Finland. Br J Audiol 1997;31:165-75.

13. Johnson CL, Paulose-Ram R, Ogden CL, et al. National Health and Nutrition Examination Survey: Analytic Guidelines, 1999-2010. National Center for Health Statistics. Vital Health Stat 2013;2:1-24.

14. National Health and Nutrition Examination Survey. Audiometry/ Tympanometry Procedures Manual 2001. Available at: http:// www.cdc.gov/nchs/data/nhanes/au.pdf. Accessed October 8, 2013.

15. Kaufman P, Alt MN, Chapman CD. Dropout Rates in the United States: 2001 (NCES 2005-046). U.S. Department of Education. National Center for Education Statistics. 2004. Washington, D.C.: U.S. Government Printing Office; 2004.

16. National Health and Nutrition Examination Survey. 1999-2000 Data Documentation, Codebook, and Frequencies. Available at: http://www.cdc.gov/nchs/nhanes/nhanes 1999-2000/DEMO.htm. Accessed May 9, 2014.

17. U.S. Department of Labor. The employment rate and beyond: alternative measures of labor underutilization. Issues in Labor Statistics. 2008. Available at: http://www.bls.gov/opub/ils/pdf/opbils67.pdf. Accessed May 14, 2014.

18. Archer KJ, Lemeshow S. Goodness-of-fit test for a logistic regression model fitted using survey sample data. Stata J. 2006; 6:97-105.
19. Helvik A-S, Krokstad S, Tambs K. Socioeconomic inequalities in hearing loss in a healthy population sample: The HUNT Study. Am J Public Health 2009;99:1376-8.

20. Hasson D, Theorell T, Westerlund H, et al. Prevalence and characteristics of hearing problems in a working and non-working Swedish population. J Epidemiol Commun Health 2010;64:453-60.

21. Hogan A, O'Loughlin K, Davis A, et al. Hearing loss and paid employment: Australian population survey findings. Int J Audiol 2009;48:117-22.

22. Cruickshanks KJ, Nondahl DM, Tweed TS, et al. Education, occupation, noise exposure history and the 10 -yr cumulative incidence of hearing impairment in older adults. Hearing Res 2010;264:3-9.

23. Jung D, Bhattacharyya N. Association of hearing loss with decreased employment and income among adults in the United States. Ann Otol Rhinol Laryngol 2012;121:771-5.

24. Agrawal Y, Platz EA, Niparko JK. Prevalence of hearing loss and differences by demographic characteristics among U.S. adults: data from the National Health and Nutrition Examination Survey, 1999-2004. Arch Intern Med 2008;168:1522.

25. Brant LJ, Fozard JL. Age changes in pure-tone hearing thresholds in a longitudinal study of normal human aging. J Acoust Soc Am 1990; 88:813-20.

26. Helzner EP, Cauley JA, Pratt SR, et al. Race and sex differences in age-related hearing loss: the Health, Aging and Body Composition Study. Journal of the American Geriatrics Society 2005;53: 2119-27.

27. Cooper JC. Health and Nutrition Examination Survey of 1971-75: Part I. Ear and race effects in hearing. J Am Acad Audiol 1994; $5: 30-6$.

28. Lin FR, Maas P, Chien W, et al. Association of skin color, race/ ethnicity, and hearing loss among adults in the USA. J Assoc Res Otolaryngol 2012;13:109-17.

29. Beckles GL, Truman BI. National Center for Chronic Disease Prevention and Health Promotion, CDC. Education and income - United States, 2009 and 2011. MMWR Surveill Summ 2013;62:9-19.

30. Fronczek P. Income, earnings, and poverty from the 2004 American Community Survey. Available at: https://www.census.gov/prod/ 2005pubs/acs-01.pdf. Accessed May 14, 2014.

31. Athar HM, Chang M-H, Hahn RA, et al. Unemployment - United States, 2006 and 2010. MMWR Surveill Summ 2013;62:27-32.

32. Kramer SE, Kapteyn TS, Houtgast T. Occupational performance: comparing normally-hearing and hearing-impaired employees using the Amsterdam Checklist for Hearing and Work. Int J Audiol 2006;45:503-12.

33. Vogel I, Verschuure H, Van der Ploeg CPB, et al. Adolescents and MP3 Players: Too Many Risks, Too Few Precautions. Pediatrics 2009;123:e953-8.

34. Pallotto EK, Kilbride HW. Perinatal outcome and later implications of intrauterine growth restriction. Clin Obstet Gynecol 2006; 49:257-69.

35. Resnick MB, Gueorguieva RV, Carter RL, et al. The impact of low birth weight, perinatal conditions, and sociodemographic factors on educational outcome in kindergarten. Pediatrics 1999;104:e74.

36. Agrawal Y, Platz EA, Niparko JK. Risk factors for hearing loss in US adults: data from the National Health and Nutrition Examination Survey, 1999 to 2002. Otol Neurotol 2009;30:139-45.

37. Cruickshanks KJ, Klein R, Klein BE, et al. Cigarette smoking and hearing loss: the epidemiology of hearing loss study. JAMA 1998;279:1715-9.

38. Fransen E, Topsakal V, Hendrickx J-J, et al. Occupational noise, smoking, and a high body mass index are risk factors for age-related hearing impairment and moderate alcohol consumption is protective: a European population-based multicenter study. $J$ Assoc Res Otolaryngol 2008;9:264-76, discussion 261-3.

39. Curhan SG, Eavey R, Wang M, et al. Body Mass Index, Waist Circumference, Physical Activity, and Risk of Hearing Loss in Women. Am J Med 2013;126:1142.e1-1142.e8. 